\title{
Panton-Valentine Leukocidin associated Staphylococcus aureus musculoskeletal infection in children. A reflective case series of long term complications
}

G. Yip, D. Davenport, T. Shah, M. Kokkinakis. Evelina London Children's Hospital, London, UK.

\section{Background}

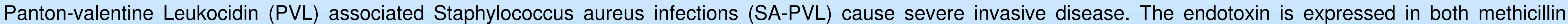

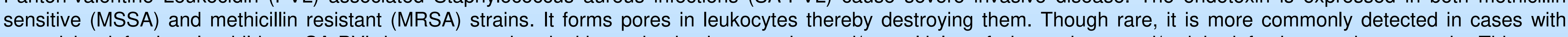

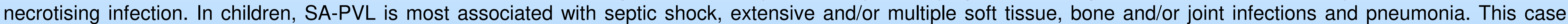
series of SA-PVL infections with musculoskeletal involvement in a paediatric population illustrate presentation, management and destructive sequelae.

\section{Cases}

\section{Case 1}

2 month old with septic shock, acute respiratory distress syndrome and leg swelling. She had femoral osteomyelitis, a subperiosteal collection and myositis, requiring surgical debridement. She subsequently developed a pathological fracture with a non-union requiring fixation and distal femoral growth arrest.

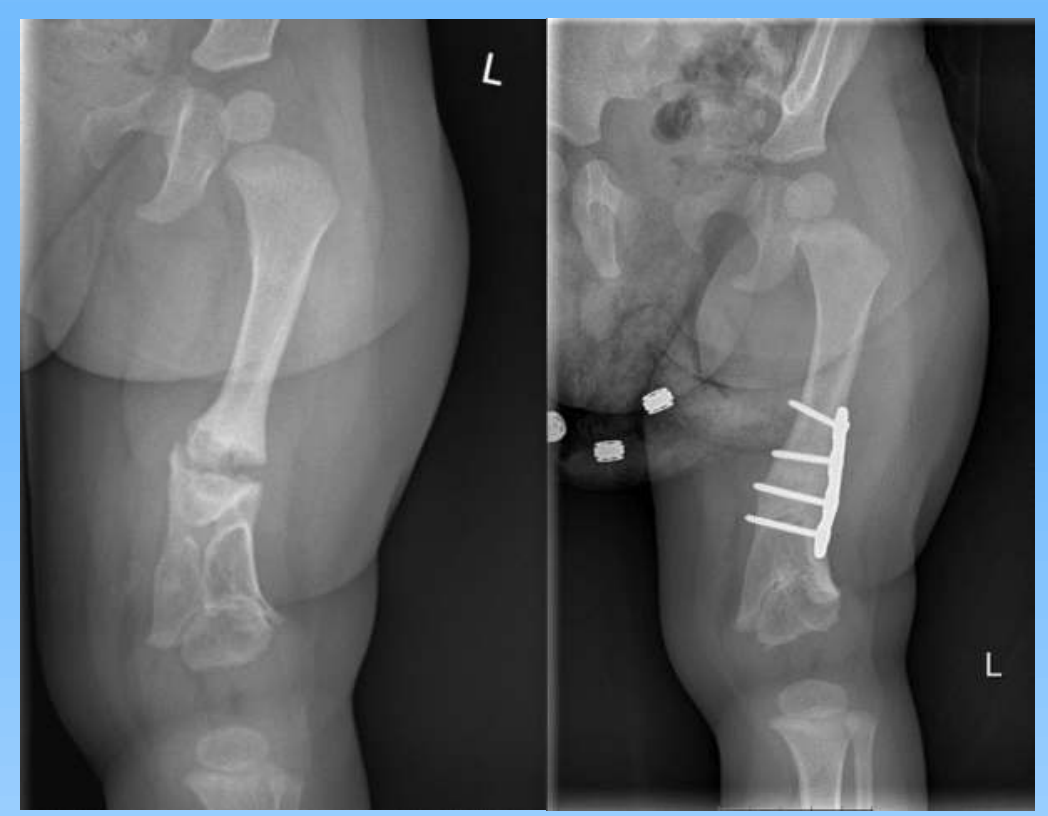

Figure 1: Left distal femoral pathological fracture and subsequent fixation with plate

\section{Case 2}

20 month old with septic shock, acute respiratory distress syndrome and acute shoulder septic arthritis. There was subsequent chronic osteomyelitis of the humerus with a pathological fracture bone loss, treated with fixation and vascularised fibula graft.

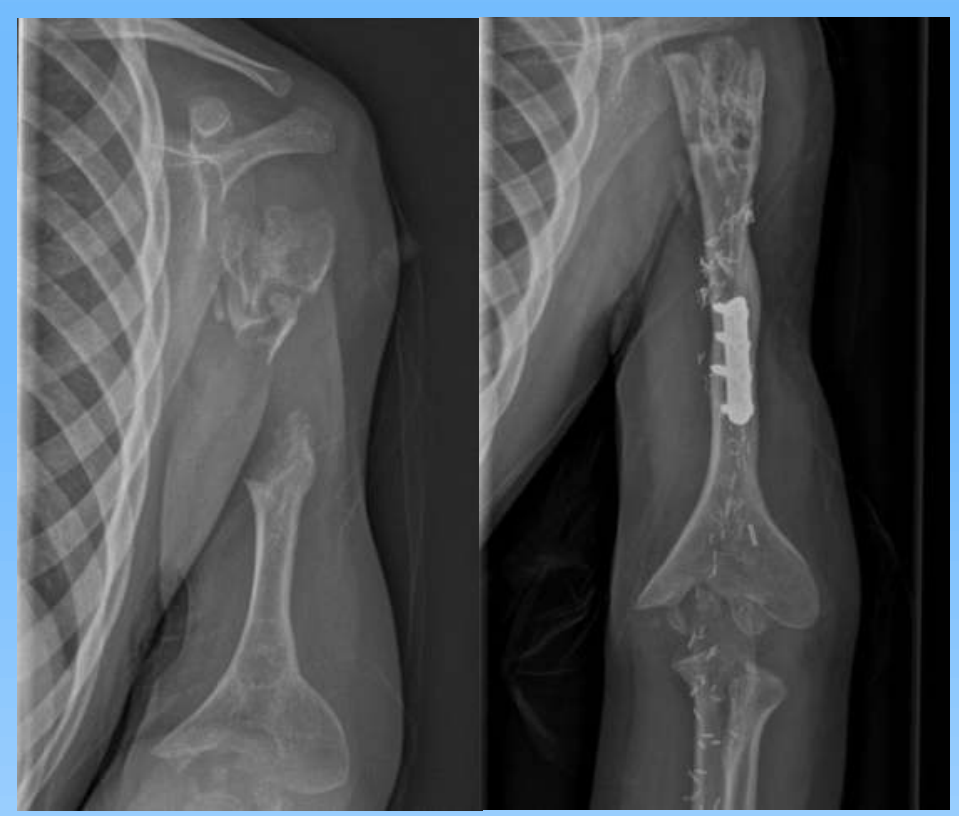

Figure 2: Left humeral pathological fracture, bone loss and subsequent fixation and vascularized fibula graft

\section{Case 3}

15 year old with acute ankle septic arthritis, subsequent chronic osteomyelitis of the distal tibia with chondrolysis and clinical arthrodesis.

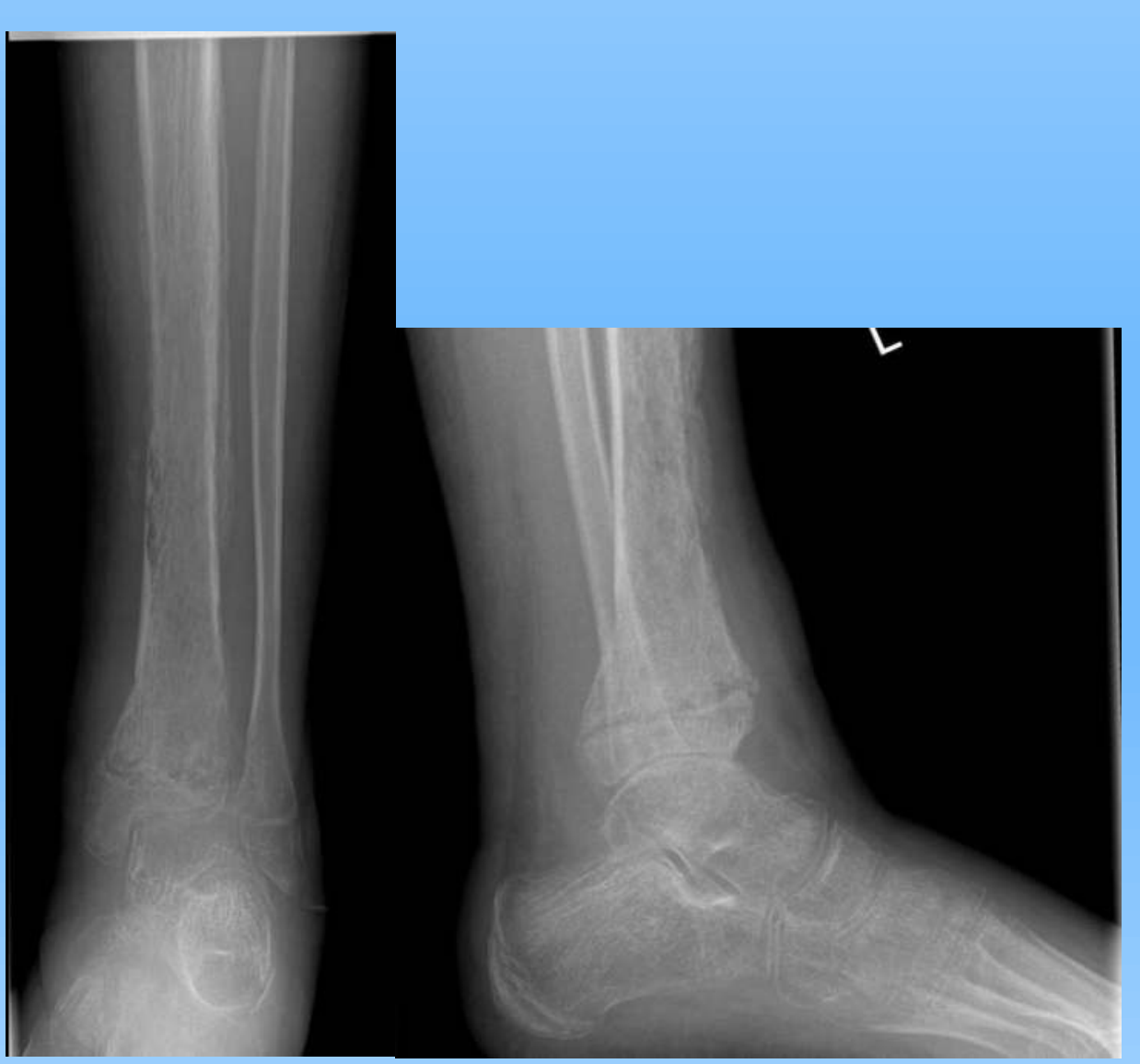

Figure 3: Ankle chondrolysis
Case 4

10 year old with right distal femoral osteomyelitis, pathological fracture and bone loss treated with external fixator and bone transport. He also had infective endocarditis and thromboemboli.

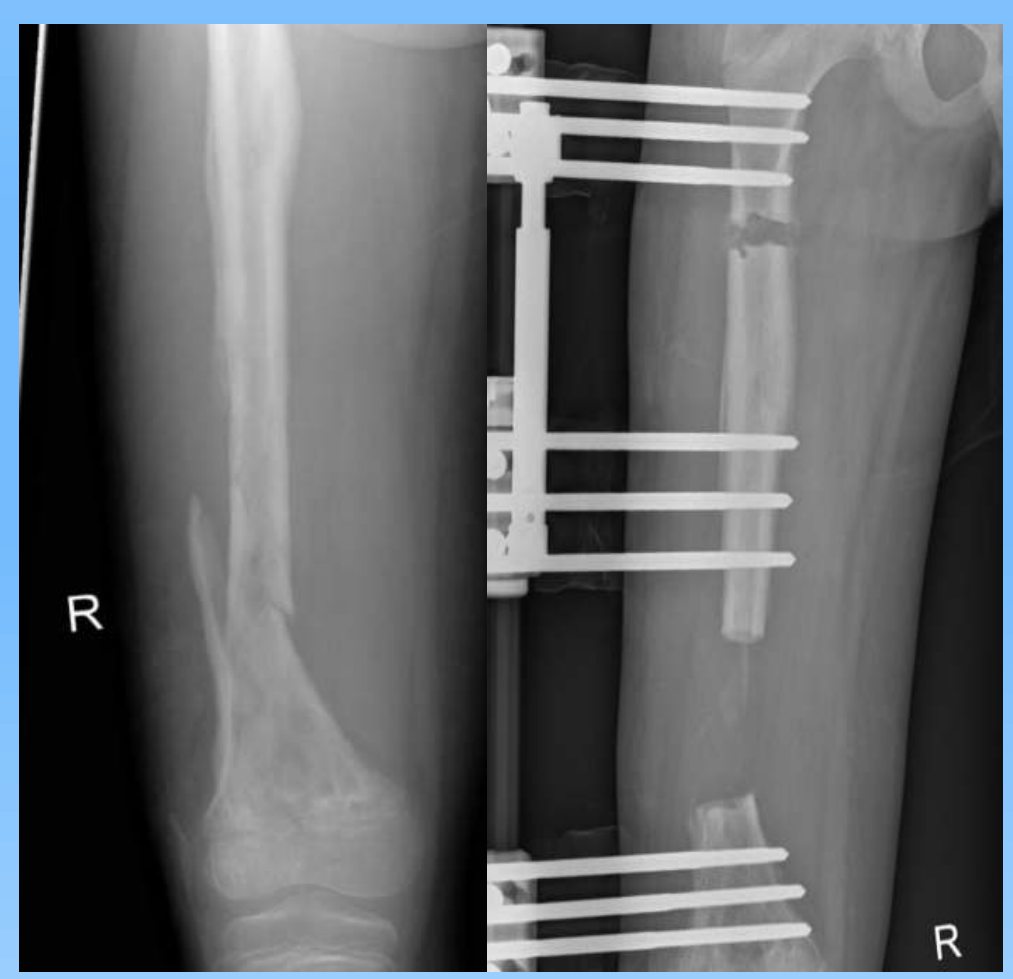

Figure 4: Distal femur pathological fracture; post debridement of sequestrum and lengthening with bone transport using external fixator

\section{Learning points/discussion}

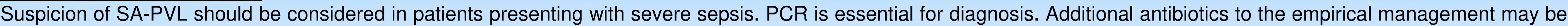

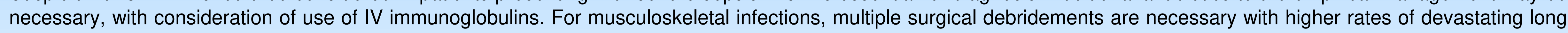

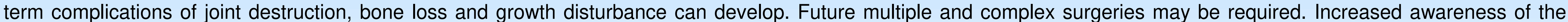
rapid onset and severe consequences of SA-PVL infections with early joint microbiology/infectious disease and surgical input are essential. 